\title{
ON IDEALLY FINITE LIE ALGEBRAS WHICH ARE LOWER SEMI-MODULAR
}

\author{
by DAVID TOWERS
}

(Received 19th October 1983)

The purpose of this paper is twofold: first to correct the statement of Theorem 1 in [4], and secondly to consider related problems in the class of ideally finite Lie algebras.

Throughout, $L$ will denote a Lie algebra over a field $K, F(L)$ will be its Frattini subalgebra and $\phi(L)$ its Frattini ideal. We will denote by $\mathfrak{X}$ the class of Lie algebras all of whose maximal subalgebras have codimension 1 in $L$. The Lie algebra with basis $\left\{u_{-1}, u_{0}, u_{1}\right\}$ and multiplication $u_{-1} u_{0}=u_{-1}, u_{-1} u_{1}=u_{0}, u_{0} u_{1}=u_{1}$ will be labelled $L_{1}(0)$.

Theorem 1 of [4] claimed that a necessary and sufficient condition for $L$ to belong to $\mathfrak{X}$ is that $L / \phi(L)=S \oplus R$, where $S$ is a simple ideal isomorphic to $L_{1}(0)$, or is $\{0\}$, and $R$ is a supersoluble ideal of $L / \phi(L)$ (possibly $\{0\}$ ). The necessity is correct, but not the sufficiency. The only problem is that $L_{1}(0)$ may not belong to $\mathfrak{X}$; when it does is described in the following lemma.

Lemma 0. $L_{1}(0) \in \mathfrak{X}$ if and only if $K$ has characteristic two, or $\sqrt{K}=\{\sqrt{k}: k \in K\} \subseteq K$.

Proof. If $K$ has characteristic two, then $F\left(L_{1}(0)\right)$ is spanned by $u_{0}$ and so all maximal subalgebras of $L_{1}(0)$ are two dimensional.

Let $S(\lambda, \mu, v)$ be the 1-dimensional subalgebra of $L_{1}(0)$ spanned by $\lambda u_{-1}+\mu u_{0}+v u_{1}$ $(\lambda, \mu, v \in K)$. Then any 1-dimensional subalgebra of $L_{1}(0)$ is of the form $S(\lambda, \mu, 0)$ or $S(\lambda, \mu, 1)$. If $\sqrt{K} \subseteq K$ then $S(\lambda, \mu, 0)$ is contained in the subalgebra spanned by $u_{0}$ and $u_{-1}$, and $S(\lambda, \mu, 1)$ is contained in that spanned by $\lambda u_{-1}+\mu u_{0}+u_{1}$ and $\alpha u_{1}-u_{-1}$ where $\alpha^{2} \lambda^{2}+2 \alpha\left(\lambda-\mu^{2}\right)+1=0$.

If $\sqrt{K} \nsubseteq K$, let $\alpha \in \sqrt{K}, \alpha \notin K$. Then, when the characteristic of $K$ is different from two, the subalgebra of $L_{1}(0)$ spanned by $\left(\alpha^{2} / 2\right) u_{1}-u_{-1}$ is maximal.

Using the above lemma and the fact that $L$ is supersoluble whenever $L / \phi(L)$ is supersoluble ([1], Theorem 6), we can correct Theorem 1 of [4] as follows.

Theorem 1. Let $L$ be a finite-dimensional Lie algebra.

(i) If $\sqrt{K} \nsubseteq K$ and $K$ has characteristic different from 2 , then $L \in \mathfrak{X}$ if and only if $L$ is supersoluble.

(ii) If $\sqrt{K} \subseteq K$ or $K$ has characteristic two, then $L \in \mathfrak{X}$ if and only if $L / \phi(L)=S \oplus R$ where $S$ is a simple ideal of $L / \phi(L)$ isomorphic to $L_{1}(0)$, or is $\{0\}$, and $R$ is a supersoluble ideal of $L / \phi(L)$ (possibly $\{0\}$ ). 
The Lie algebra $L$ is lower semi-modular if, whenever $U, V$ are distinct subalgebras of $L$ both of which are maximal in the subalgebra $W$ of $L$, then $U \cap V$ is maximal in both $U$ and $V$. Recall that $L$ is ideally finite if every element of $L$ lies in a finite-dimensional ideal of $L$. The reader is referred to [3] for any results on ideally finite Lie algebras which are used. Also following Stewart in [3] we call $L$ hypercyclic if it has an ascending series of ideals $\left(L_{\alpha}\right)_{\alpha \leqq \sigma}$ such that $\operatorname{dim} L_{\alpha+1} / L_{\alpha}=1$ for all $\alpha<\sigma$.

Our main result is the following.

Theorem 2. Let $L$ be an ideally finite Lie algebra. Then the following are equivalent.

(i) $L$ is locally supersoluble.

(ii) $L \in \mathfrak{X}$ and is locally soluble.

(iii) $L$ is hypercyclic.

(iv) $L$ is locally soluble and lower semi-modular.

Proof. (i) $\Rightarrow$ (ii): Suppose that $L$ is supersoluble; then $L$ is clearly locally soluble. Let $M$ be a maximal subalgebra of $L$ and pick $x \notin M$. Then there is an ideal $I$ of $L$ with $x \in I$ and $\operatorname{dim} I<\infty$. Now $L=M+I$, so $M$ has finite codimension in $L$. Put $C=C_{L}(I)=$ $\{x \in L: x I=0\}$, so that $\operatorname{dim} L / C<\infty$. If $C \subseteq M$, then $M / C$ is a maximal subalgebra of $L / C$, which is supersoluble, and so $M$ has codimension 1 in $L$. So suppose that $C \nsubseteq M$, and hence that $L=C+M$. But $C \cap M$ is an ideal of $L$, and so $L / C=(C+M) / C \cong M / C \cap M$. It follows that $C \cap M$ has finite codimension in $M$ and hence in $L$. Thus $M / C \cap M$ is a maximal subalgebra of $L / C \cap M$, which is soluble, and again $M$ has codimension 1 in $L$.

(ii) $\Rightarrow$ (iii): Suppose that $L \in \mathfrak{X}$ and is locally soluble. We need only prove that the minimal ideals of $L$ are 1-dimensional. Clearly we may assume that the centre of $L$ is trivial, and hence that $L$ is residually finite. Let $A$ be a minimal ideal of $L$. Then $A$ is finite dimensional and so, by Lemma 3.4 of [3], there is an ideal $K$ of $L$ with $\operatorname{dim} L / K<\infty$ and $K \cap A=\{0\}$. Now all maximal subalgebras of $L / K$ have codimension 1 in $L / K$, and $L / K$ is soluble. Hence $L / K$ is supersoluble. But $A \cong(A+K) / K$, which is a minimal ideal of $L / K$, and so is 1 -dimensional.

(iii) $\Rightarrow$ (i): Let $L$ be hypercyclic and let $U$ be a finitely generated subalgebra of $L$. Then $U \subseteq I$ where $I$ is a finite-dimensional ideal of $L$. Clearly $I$, and hence $A$, is supersoluble.

(ii) $\Rightarrow$ (iv): Let $L \in \mathfrak{X}$ be locally soluble, and let $U, V$ be distinct subalgebras of $L$, both of which are maximal in the subalgebra $W$ of $L$. Since (ii) is equivalent to (i), and hypothesis (i) is subalgebra closed, we may assume that $W=L$. Then $U, V$ have codimension 1 in $L$, so $L=U+V$. Clearly, $U \cap V$ has codimension 1 in both $U$ and $V$, and so $L$ is lower semi-modular.

(iv) $\Rightarrow$ (i). Let $L$ be locally soluble and lower semi-modular, and let $U$ be a finitely generated subalgebra of $L$. Then $U$ is a finite-dimensional soluble Lie algebra which is lower semi-modular. It follows from Lemma 5 of [2] that $U$ is supersoluble.

We can deduce from the above result the following analogue of Theorem 6 of [1].

Corollary 3. Let $L$ be an ideally finite Lie algebra, let $A$ be an ideal of $L$ with $A \subseteq \phi(L)$ (the Frattini ideal of $L$ ), and suppose that $L / A$ is hypercyclic. Then $L$ is hypercyclic.

Proof. We have that $L / A$ is locally soluble, and $A$ is locally nilpotent (see [3]), so $L$ 
is locally soluble. Furthermore, all maximal subalgebras of $L$ contain $A$, and so have codimension 1 in $L$, by Theorem 2. It follows from Theorem 2 again that $L$ is hypercyclic.

\section{REFERENCES}

1. D. W. Barnes, On the cohomology of soluble Lie algebras, Math. Z. 101 (1967) 343-349.

2. A. G. Gein, Semimodular Lie algebras, Siberian Math. J. 17 (1976), 189-193.

3. I. N. StewART, Lie algebras generated by finite-dimensional ideals (Pitman 1975).

4. D. A. Towers, Lie algebras, all of whose maximal subalgebras have codimension 1, Proc. Edinburgh Math. Soc. 24 (1981), 217-219.

Department of Mathematics

UNIVERSITY OF LANCASTER

LANCASTER

LA1 4YL 